\title{
Looking after silent coronary artery disease in diabetic patients. When and how?
}

\author{
Paul Valensi
}

Published online: 17 September 2011

(C) Springer Science+Business Media, LLC 2011

Myocardial infarction is often silent in diabetic patients, probably as often as it is symptomatic [1]. Major arguments stand in favor of screening patients for silent myocardial ischemia (SMI), including the predictive value of SMI for cardiovascular events above and beyond risk scores [2], with the poorest prognosis in patients with extended ischemia and/or coronary stenoses [3]. However, a controversy has recently emerged with regard to the usefulness of screening, based on the improvement of cardiovascular prognosis in the diabetic population, feasibility and costeffectiveness concerns, and the lack of evidence for a benefit of screening on outcomes. In fact, treatment intensification targeting the major risk factors reduces by $50 \%$ the rate of cardiovascular events but allows a high residual risk which may be related to silent coronary artery disease (CAD). Regarding the second argument, given the huge number of diabetic patients, it is neither possible nor logical to screen all of them for SMI. Thus, a careful patient selection based on coronary risk stratification is mandatory. In this issue of Endocrine, the article by Gazzaruso et al. [4] addresses the question of the effectiveness of screening for CAD. The authors screened 299 consecutive men with newly diagnosed type 2 diabetes (T2D) without heart or peripheral artery disease. SMI was detected by an ECG stress test with a subsequent confirmation based mostly upon defects on stress myocardial

\footnotetext{
P. Valensi

Department of Endocrinology-Diabetology-Nutrition AP-HP, Jean Verdier Hospital, Paris-Nord University, CRNH-IdF,

Bondy, France

P. Valensi $(\bowtie)$

Service d'Endocrinologie Diabétologie Nutrition, Hôpital Jean Verdier, Avenue du 14, Juillet, Bondy 93140, France

e-mail: paul.valensi@jvr.aphp.fr
}

scintigraphy, and CAD was assessed on angiography in the patients with confirmed SMI. SMI prevalence was around $27 \%$, and the prevalence of coronary stenoses $\geq 50 \%$ of the lumen was around $25 \%$. In another Italian study performed in patients with the same characteristics, the rate of SMI according to the ECG stress test was quite similar in men [5]. In the MiSAD study also performed in Italy, which included patients with long-standing diabetes, SMI as defined by the same two tests was found in only around $8 \%$ of men [6]. In several studies, patients with long-standing diabetes and other risk factors, many of whom also had peripheral artery disease and nephropathy, were all screened by stress myocardial scintigraphy or stress echocardiography. The rate of SMI was usually $20-35 \%$, and CAD was found in one- to two-thirds of those with SMI, i.e., in $10-15 \%$ of the overall cohorts [3]. Therefore, the rates of SMI in newly diagnosed T2Ds are surprisingly high. This suggests that some patients had previously undiagnosed diabetes and is also consistent with the "common soil" hypothesis for T2D and CAD. The fact that SMI or CAD is not more prevalent in higher-risk patients with long-standing diabetes might result from the beneficial effects of antidiabetic treatment and treatment risk factors. As expected, screening without subsequent therapeutic adaptation, including or not including coronary revascularization, does not appear to affect overall outcomes [7]. One pilot randomized study and one retrospective study suggest that revascularization may be beneficial to patients with SMI [3]. However, the benefit of stable CAD has recently been questioned. Therefore, a major challenge is to identify patients with severe CAD who are most likely to benefit from coronary artery bypass grafting, as shown in the BARI-2D trial. Improving the cost-effectiveness of screening will allow us to detect such patients while performing fewer investigations in a lower 
number of patients. In the Gazzaruso et al. study [4], only 23 patients had a three-vessel coronary disease $(<8 \%$ of the study population). This percentage was even lower in other comparable studies. How shall we identify these patients? In our cohort of more than 700 T2Ds, both ADA and French guidelines were able to predict SMI, but prediction was improved through the consideration of male gender and the presence of retinopathy (paper submitted for publication). Some markers of $\mathrm{CAD}$, such as abnormalities on resting echocardiogram, a high coronary calcification score, low levels of serum L-selectin, and slightly increased plasma pro-BNP levels, may be helpful [3]. Interestingly, adding erectile dysfunction (ED) to the list of risk factors seems to increase the sensitivity of the ADA guidelines from 62 to $89 \%$, without a significant variation in specificity [4]. Coronary endothelial function was shown to be impaired in patients with SMI, but angiographically normal coronary arteries [8]. Endothelial dysfunction is probably the link between ED and CAD. An accurate process should also improve cost-effectiveness. In the Gazzaruso et al. study [4], stress scintigraphy, performed as a second-step test, was confirmatory of a positive ECG stress test in $94 \%$ of the cases (a very high rate compared to other studies [9]), and coronary stenoses were found in all but one of these patients. This suggests that the ECG stress test is highly predictive of CAD, and that a second test is probably unnecessary in men with newly diagnosed T2D. On the contrary, in the MiSAD study, the more restrictive criterion defined by a positive response to both tests reduced the prevalence of SMI from 13.7 to $7.9 \%$ [6]. In our center, the agreement between the two tests is low in men and even lower in women, many patients are detected with SMI only upon defects on scintigraphy, and combining a myocardial scintigraphy with a maximal ECG stress test is effective in detecting more patients with CAD [9]. The test performance depends on risk level in accordance with Bayes' theorem, and needs to be further evaluated separately in men and women. Detecting SMI using a twostep process may ignore some patients with CAD. The improvement of CAD risk prediction by $\mathrm{ED}$ on top of risk factors may be confirmed in another study that includes stress scintigraphy or echocardiography in all patients.

Finally, patients with both severe SMI and CAD are the most likely to benefit from specific anti-ischemic treatments and, probably, revascularization beyond the effective control of risk factors. Therefore, screening for SMI should be proposed to patients who have long-standing T2D are at high risk as defined by elevated risk scores, and have micro- and macrovascular complications, ED, and, eventually, a high calcium coronary score, once they are at goal for risk factors.

\section{References}

1. P. Valensi, L. Lorgis, Y. Cottin, Prevalence, incidence, predictive factors and prognosis of silent myocardial infarction: a review of the literature. Arch. Cardiovasc. Dis. 104, 178-188 (2011)

2. E. Cosson, M.T. Nguyen, B. Chanu, I. Banu, S. Chiheb, C. Balta, K. Takbou, P. Valensi, Cardiovascular risk prediction is improved by adding asymptomatic coronary status to routine risk assessment in type 2 diabetic patients. Diabetes Care 34, 2101-2107 (2011)

3. P. Valensi, E. Cosson, It is not yet the time to stop screening diabetic patients for silent myocardial ischaemia. Diabetes Metab. 36, 91-96 (2009)

4. Gazzaruso C, Coppola A, Montalcini T, Valenti C, Garzaniti A, Pelissero G, Salvucci F, Gallotti P, Pujia A, Falcone C, Solerte SB, Giustina A: Erectile dysfunction can improve the effectiveness of the current guidelines for the screening for asymptomatic coronary artery disease in diabetes. Endocrine (2011). doi:10.1007/s12020011-9523-9

5. P. Fornengo, A. Bosio, G. Epifani, O. Pallisco, A. Mancuso, C. Pascale, Prevalence of silent myocardial ischaemia in new-onset middle-aged type 2 diabetic patients without other cardiovascular risk factors. Diabet. Med. 23, 775-779 (2006)

6. Milan Study on Atherosclerosis and Diabetes (MiSAD) Group, Prevalence of unrecognized silent myocardial ischemia and its association with atherosclerotic risk factors in noninsulin-dependent diabetes mellitus. Am. J. Cardiol. 79, 134-139 (1997)

7. L.H. Young, F.J. Wackers, D.A. Chyun, J.A. Davey, E.J. Barrett, R. Taillefer, G.V. Heller, A.E. Iskandrian, S.D. Wittlin, N. Filipchuk, R.E. Ratner, S.E. Inzucchi, Cardiac outcomes after screening for asymptomatic coronary artery disease in patients with type 2 diabetes: the DIAD study: a randomized controlled trial. JAMA 301, 1547-1555 (2009)

8. A. Nitenberg, P. Valensi, R. Sachs, M. Dali, E. Aptecar, J.R. Attali, Impairment of coronary vascular reserve and ACh-induced coronary vasodilation in diabetic patients with angiographically normal coronary arteries and normal left ventricular systolic function. Diabetes 42, 1017-1025 (1993)

9. E. Cosson, F. Paycha, J. Paries, S. Cattan, A. Ramadan, D. Meddah, J.R. Attali, P. Valensi, Detecting silent coronary stenoses and stratifying cardiac risk in patients with diabetes: ECG stress test or exercise myocardial scintigraphy? Diabet. Med. 21, 342-348 (2004) 